\title{
Towards a more open and transparent plant pathology research
}

\section{Emerson M. Del Ponte ${ }^{1}$}

Published online: 14 August 2020

(C) Sociedade Brasileira de Fitopatologia 2020

I am thrilled to announce new alliances and changes to our editorial board structure and peer-review workflow. These will represent important step towards an inevitable move in the near future to a more stringent data sharing policy, in line with other Nature Research and BMC journals. Our data sharing policy has been stuck in category 1 since they were established a few years ago. We have made a decision to adopt category 3 effective mid-2020, which will require from authors a Data Availability Statement (DAS). Instead of the data associated with a publication available on request (category 1 , no DAS), authors are strongly encouraged to deposit the data in a list of recommended repositories. The inclusion of DAS, where the author must state if and how their research data are made available, is increasingly being encouraged or mandated by publishers nowadays.

The practice of requesting the deposit of data in public repositories is not new in plant pathological scholarly publications, but has been limited to certain types of data. The provision of the accession numbers of nucleotide data in repositories is a well-established requirement by editors and reviewers of journals including Tropical Plant Pathology. Nevertheless, sharing of general data in scholarly peerreviewed work in the field of plant pathology is very rare (Sparks et al. 2020). There is no much reason why the request cannot be extended to other data than molecular. The availability of several discipline-specific archives or generalpurpose open repositories has facilitated considerable archiving and licensing of the data and provision of digital object identifiers (DOIs) for enhanced discoverability and credit, although the possibility of sharing raw data as supplemental material has been around for a long time.

The benefits from making data and any other outcome of the research available, such as computer code for statistical analysis, have been advertised and are multifold. The

Emerson M. Del Ponte

delponte@ufv.br

1 UFV Departamento de Fitopatologia, Universidade Federal de Viçosa (UFV), Viçosa, Brazil submission of the data, as well as the scripts used for the analysis, along with the manuscript, contributes to transparency of science. It potentially adds more value to the peerreview process because reviewers may check more closely and reproduce the analysis, providing more useful advice with the data in hand. After the manuscript is accepted, the proper documentation and storage of data and codes, besides constituting a citable unit themselves - so that one gains the deserved credit for all the research work, extends the value of the investment in research and open door for collaboration due to visibility. In fact, funding bodies and research institutes are establishing data sharing policies for funded projects that consider publicly research data as a public good that should be openly available. Given the role that journals play in disseminating research results that rely on data to support the findings, facilitating access to data should be a cornerstone. Finally, sharing data may improve paper's citation rate. Studies on the effect of data sharing policies adopted by journals are very encouraging as suggested by the analysis of 500+ scientific articles in BMC and PLOS journals that showed an increase of up to $25 \%$ in citations of articles with category 3 DAS compared to basic categories (Colavizza et al. 2020).

We acknowledge possible difficulties and barriers for the open sharing of plant disease-related data. Author's resistance, or other factors that inhibit data sharing, could impact the submission rate to journals that mandate data sharing, especially if similar policy is not adopted by other journals of the field, which is the current scenario. Perhaps less commonly, some data cannot be released publicly due to institutional policies or embargo. My view of the current scenario is that the practice of data sharing in the general field of plant pathology is hindered by the fact that (a) openness culture is very incipient and benefits from data sharing are partially overshadowed by a general fear of being scooped and (b) support or training on how to prepare, document, and archive the data in a way that facilitates reproducibility is clearly lacking in our scientific community.

To tackle these challenges and provide support for our new data policy, we established a partnership with Open Plant 
Pathology (OPP, http://www.openplantpathology.org), an initiative that supports and promotes the spread of open, transparent, and reproducible practices in our field. We created a special team led by Dr. Adam Sparks (University of Southern Queensland) who is taking over as Reproducibility Editor. The mission of this new team is to promote the new data policy and support authors who agree to make their work as reproducible as possible, in which shared data and computer codes are the keystones. Alongside, educational campaigns in the form of online tutorials and workshops on how to engage in reproducible practices have been one of the goals and will be intensified by Open Plant Pathology. The future ahead is exciting and hopefully similar efforts are embraced by other plant pathology journals in order to enhance transparency and scientific credibility of our discipline.

\section{References}

Colavizza G, Hrynaszkiewicz I, Staden I, Whitaker K, McGillivray B (2020) The citation advantage of linking publications to research data. PLoS One 15:e230416

Sparks AH, Del Ponte EM, Alves KS, Foster Z, Grünwald N (2020) Compendium of $\mathrm{R}$ code and data for 'Status and Best Practices for Reproducible Research In Plant Pathology’. Accessed 21 Apr 2020. Online at https://doi.org/10.5281/zenodo.1250664

Publisher's note Springer Nature remains neutral with regard to jurisdictional claims in published maps and institutional affiliations. 